\title{
Tumor maligno da bainha perineural retrobulbar e intracraniano em cão: relato de caso
}

[Malignant neoplasm of the perineural sheath retrobulbar and intracranial in dog: case report]

\author{
C.G.G. Bordini ${ }^{1,2}$, E.O. Silva ${ }^{1,3}$, K.T. Green ${ }^{4}$, M.S.B. Souza $a^{5}$, G.W. Santis ${ }^{5}$, M.S. Zanutto ${ }^{5}$ \\ ${ }^{1}$ Aluno de pós-graduação - Universidade Estadual de Londrina - Londrina, PR \\ ${ }^{2}$ Universidade Norte do Paraná - Unidade Piza e Arapongas, PR \\ ${ }^{3}$ Universidade do Oeste Paulista - Presidente Prudente, SP \\ ${ }^{4}$ The Ministry of Agriculture e Fisheries- Veterinary Services Division, Jamaica \\ ${ }^{5}$ Universidade Estadual de Londrina - Londrina, PR
}

\section{RESUMO}

Os tumores da bainha perineural são pouco frequentes em animais domésticos. Relata-se o caso de um cão, sem raça definida, de cinco anos, fêmea, com histórico de aumento de volume em região retrobulbar do globo ocular direito (GOD) havia três semanas. Ao exame clínico, constatou-se a presença de uma neoformação retrobulbar direita com deslocamento do globo ocular cranialmente. Aos exames realizados durante o internamento, não foi possível localizar a origem e a extensão da neoformação. $\mathrm{O}$ animal veio a óbito por parada cardiorrespiratória e foi encaminhado para a necropsia. À avaliação macroscópica, observou-se neoformação esbranquiçada fixada à base do crânio em região selar, com possível origem no terceiro (III) par de nervos cranianos, a qual se infiltrava no encéfalo na altura do hipotálamo, estendia-se caudalmente em direção ao tronco encefálico e cranialmente à órbita direita, comprimindo, assim, o GOD. Microscopicamente consistia de feixes curtos entrelaçados ou enovelados de células fusiformes com pleomorfismo discreto a moderado, alternando-se a áreas de necrose multifocalmente, compatível com tumor da bainha perineural. Ao exame imuno-histoquímico, apresentou marcação fraca para S100 e GFAP e marcação positiva para vimentina, o que indica caráter maligno.

Palavras-chave: cão, neoplasia maligna, tumor da bainha perineural, bradicardia, síndrome de Horner

\begin{abstract}
Tumors of the perineural sheath are uncommon in domestic animals. We report the case of a 5-year-old female dog with a history of increased volume in the retrobulbar region of the right eye (RE) three weeks ago. The clinical examination revealed a presence of a right retrobulbar neoformation with cranial ocular globe displacement. In the examinations carried out during hospitalization, it was not possible to locate a source and an extension of the neoformation. The animal died of cardiorespiratory arrest and was referred to an autopsy. The macroscopic evaluation revealed a whitish neoformation fixated to the base of the skull in a seal region, with a possible non-III origin of cranial nerves, infiltrating nonencephalon at the height of the hypothalamus, extending caudally towards the brainstem and cranially to the orbit right, compressing RE. Microscopically it consisted of short bundles intertwined or enovelados of spindle cells with discrete to moderate pleomorphism, alternating to areas of multifocal necrosis, compatible with tumor of the perineural sheath. Immunohistochemical examination showed weak marking for S100 and GFAP and positive marking for vimentin, indicating malignancy.
\end{abstract}

Keywords: dog, malignant neoplasm, perineural sheath tumor, bradycardia, Horner's syndrome

\section{INTRODUÇÃO}

Os tumores da bainha dos nervos periféricos originam-se tanto das células de Schwann como dos fibroblastos perineurais, ou de ambos (Jones

Recebido em 17 de outubro de 2017

Aceito em 12 de abril de 2018

E-mail: carol_grano@hotmail.com et al., 1995; Chijiwa et al., 2004; Viott et al., 2007; Araújo et al., 2008; Fernandez e Bernardini, 2010; Silva et al., 2012).

Os principais tumores de bainha perineural se originam das células de Schwann, por isso existe 
uma controvérsia sobre a nomenclatura, embora classicamente os schwannomas referem-se a tumores com proliferação homogênea das células de Schwann, enquanto os neurofibromas apresentam origem nas células de Schwann e incorporam componentes nervosos periféricos não neoplásicos (células perineurais e/ou fibroblastos). Os tumores malignos apresentam origens similares, no entanto ocorrem mutações em genes supressores tumorais e a malignidade pode ainda ser constatada pela infiltração dos tecidos locais, invadindo além do perineuro (Rodriguez et al., 2013).

As neoplasias de bainha são comuns em seres humanos e menos frequentes em animais domésticos, sendo sua maior ocorrência em bovinos e caninos (Jones et al., 1995; Kuamura et al., 1998; Stoica et al., 2001; LeCouter e Withrow, 2007; Viott et al., 2007; Silva et al., 2012). Em cães, ocorrem predominantemente nos nervos do plexo braquial, sendo rara sua ocorrência nos nervos cranianos e em outros nervos periféricos (Jones et al., 1995). O estudo de Attali-Soussay e colaboradores (2001) analisou 25 casos de neoplasias orbitárias em cães, e apenas um paciente relatado apresentava tumor de bainha.

Em seres humanos, as características clínicas e histomorfológicas são utilizadas para a distinção de padrões e subclassificações dos tumores de bainha, o que não ocorre na medicina veterinária, os quais são referidos como tumor da bainha perineural ou schwannoma (Stoica et al., 2001; LeCouter e Withrow, 2007; Fernandez e Bernardini, 2010; Silva et al., 2012), podendo ser benignos ou malignos (Stoica et al., 2001; Chijiway et al., 2004; Zachary, 2009).

Os tumores benignos geralmente são massas lobuladas de tamanhos variáveis, encapsuladas, com aspecto globoide, enquanto que os tumores malignos possuem formas morfológicas variadas, sendo geralmente não encapsulados e infiltrativos (Stoica et al., 2001; LeCouter e Withrow, 2007).

Em cães, o plexo braquial é mais acometido, mas pode afetar o plexo lombossacral e os nervos cranianos (Jones et al., 1995, Chijiway et al., 2004; Viott et al., 2007; LeCouter e Withrow, 2007). Em seres humanos, o nervo craniano mais acometido é o oitavo par, ao contrário do que ocorre na espécie canina, na qual o quinto par é o mais afetado (Viott et al., 2007; Ottinger et al. 2009; Zachary; 2009; Fernandez e Bernardini, 2010).

Devido aos poucos relatos, o presente trabalho tem como objetivo descrever os sinais clínicos e as alterações patológicas de um caso de tumor maligno de bainha perineural de localização retrobulbar e intracraniana em cão.

\section{RELATO DE CASO}

Foi atendido um paciente da espécie canina, sem raça definida, fêmea, de cinco anos de idade, $25 \mathrm{~kg}$, devido a aumento de volume no globo ocular direito (GOD) havia três semanas.

Ao exame físico, foram detectados bradicardia (78bpm), desidratação leve $(5 \%)$ e aumento de volume em região retrobulbar direita, com deslocamento do globo ocular cranialmente. O animal apresentava ainda síndrome de Horner em GOD e uveíte bilateralmente.

Ao hemograma, observou-se linfopenia, enquanto os exames bioquímicos estavam dentro dos valores de referência para a espécie. Foram realizadas radiografias do crânio, nas quais não foram visibilizados sinais de comprometimento ósseo da região ocular direita. Em radiografias torácicas, não havia sinais radiográficos de metástase pulmonar.

Ao exame ultrassonográfico, observou-se, em região retrobulbar de GOD, a presença de massa com limites maldefinidos, sem sinais de acometimento do globo. No exame de Doppler, verificou-se baixa vascularização. Não foi possível determinar a extensão e os limites da massa.

Durante o período de internamento, o animal permaneceu bradicárdico e, quando a frequência cardíaca aferida encontrava-se abaixo de 60bpm, era realizada atropina $(0,044 \mathrm{mg} / \mathrm{kg})$ por via $\mathrm{SC}$ ou IV. No quinto dia de internação, o animal começou a apresentar dificuldade de deglutição. Foram discutidas as opções de tratamento e qualidade de vida com os proprietários e estes optaram pelo procedimento cirúrgico para a excisão da massa; caso não fosse possível reduzir a compressão ou retirar toda a massa, optariam pela eutanásia. Contudo, na manhã do procedimento, o animal apresentou parada cardiorrespiratória e veio a óbito. 
Macroscopicamente foi observada neoformação esbranquiçada caudalmente ao quiasma óptico, a qual recobria o assoalho do crânio em região suprasselar, com limites maldefinidos, infiltrava no sistema nervoso na altura do hipotálamo e estendia-se caudalmente em direção ao tronco encefálico e craniolateralmente à órbita direita, comprimindo o GOD (Fig. 1). A neoformação estendia-se ainda ventralmente, destruindo $\mathrm{o}$ tecido ósseo e infiltrando-se no palato duro (Fig. 2).

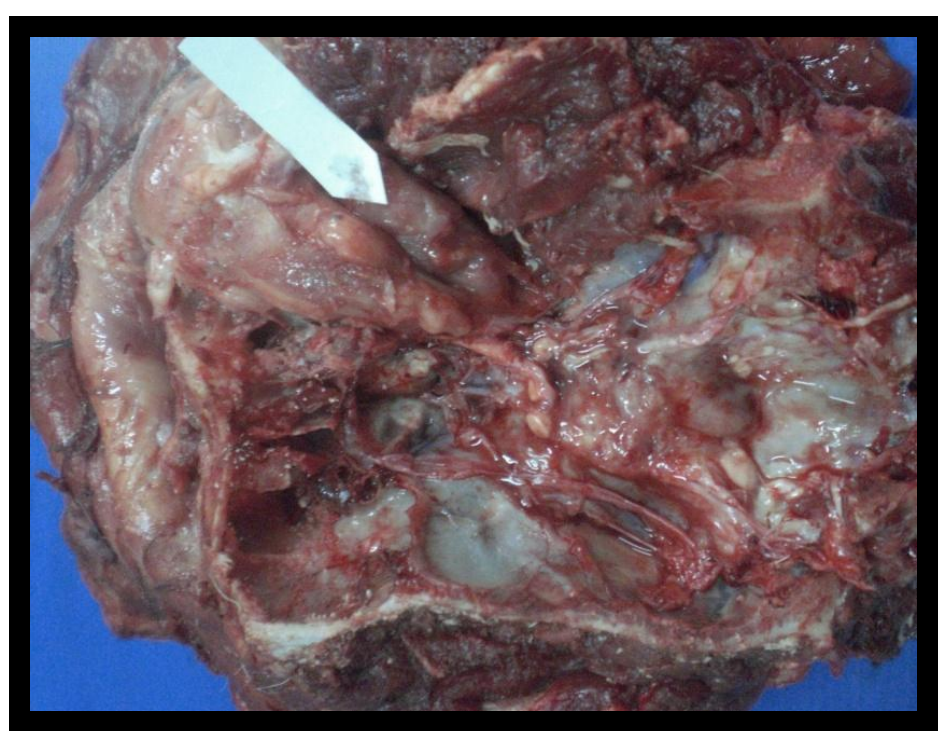

Figura 1. Cão, interior do neurocrânio após remoção do encéfalo, vista caudorrostral. Tumor maligno da bainha perineural intracraniano, de coloração esbranquiçada e consistência moderadamente friável em região suprasselar, o qual se estende rostralmente para a região retro e laterobulbar, adentra a órbita direita (marcação em papel branco) e caudalmente acompanha o tronco encefálico (removido), recobrindo a base do crânio do lado direito. Fonte: Arquivo pessoal.

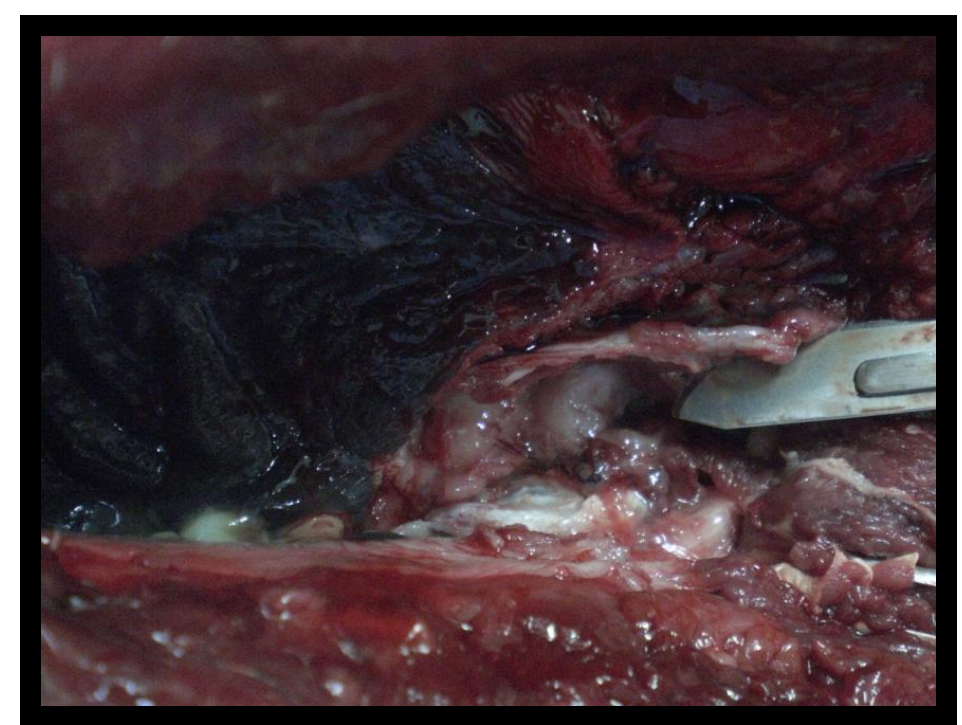

Figura 2. Cão, cavidade oral, palato duro, visão ventrodorsal. No interior da cavidade oral, observa-se palato duro pigmentado (canto superior esquerdo) e área de proliferação neoplásica (tumor maligno da bainha perineural intracraniano) indicada pela ponta da lâmina do bisturi, irregular, esbranquiçada, destruindo o tecido ósseo, vinda do interior do crânio, a partir de infiltração da região selar. Fonte: Arquivo pessoal. 
Microscopicamente, na coloração de hematoxilina-eosina, observou-se a presença de feixes curtos entrelaçados, enovelados ou em paliçada de células fusiformes com pleomorfismo discreto a moderado, com infiltrado inflamatório mononuclear de permeio e alternando-se a áreas de necrose multifocalmente (Fig. 3A e B). Ao exame imuno-histoquímico, apresentou marcação fraca para S100 e GFAP, positiva para vimentina, negativa para lisozima e negativa para citoqueratina (Fig. 3C e D).

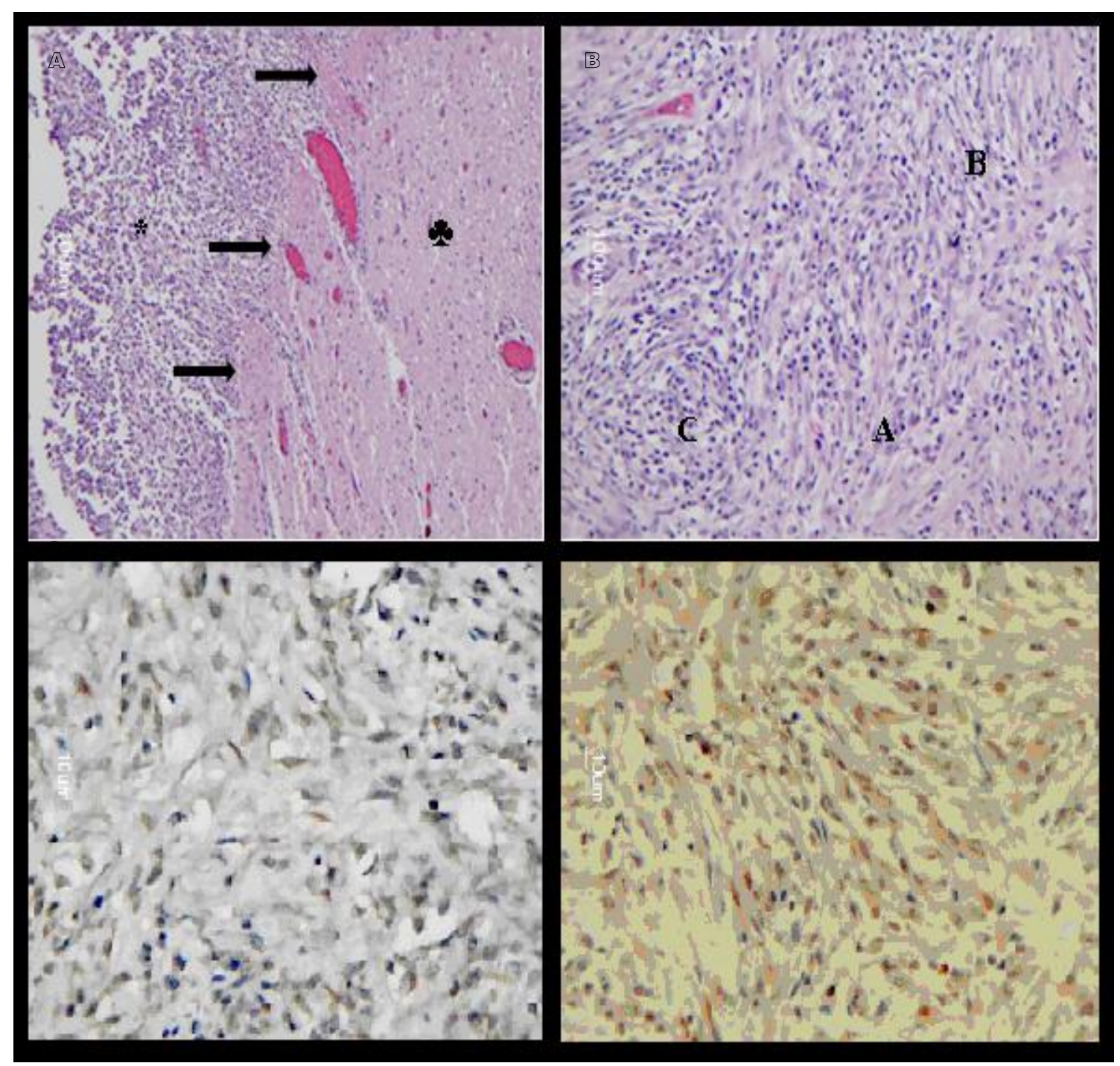

Figura 3. Fotomicroscopia digital de tumor maligno da bainha perineural intracraniano retrobulbar canino. A- Sistema nervoso central. Infiltração neoplásica $(*)$ no tecido nervoso (*) invadindo a região de hipocampo (setas). Hematoxilina-eosina, barra $100 \mu \mathrm{m}$. B- Detalhamento do padrão histológico da neoformação, com presença de células fusiformes entrelaçadas em feixes curtos (A), em paliçada (B) e enovelando-se (C). Hematoxilina-eosina, barra $100 \mu \mathrm{m}$. C- Aplicação de técnica imuno-histoquímica no tecido neoplásico onde se observa marcação fraca, citoplasmática, pelo anticorpo anti-GFAP (glial fibril aracid protein) nas células neoplásicas. Técnica do polímero, DAB, barra $10 \mu \mathrm{m}$. D- Aplicação de técnica imuno-histoquímica no tecido neoplásico, com destaque à imunomarcação citoplasmática para o anticorpo anti-S100 nas células neoplásicas. Técnica do polímero, DAB, barra $10 \mu \mathrm{m}$. Fonte: Arquivo pessoal. 


\section{DISCUSSÃO}

Os tumores de nervos periféricos são incomuns em cães, não havendo predisposição racial ou sexual, e ocorrem geralmente em animais com cinco a 12 anos, com idade média de oito anos (Jones et al., 1995; LeCouter e Withrow; 2007; Viott et al., 2007; Araújo et al., 2008; Tammenhain et al., 2008; Fernandez e Bernardini, 2010). O paciente em questão estava dentro dessa faixa etária, entretanto no limite inferior da faixa descrita. Já foi relatada predisposição em animais da raça Golden Retriever (Goldschmit e Hendrick, 2002).

Os tumores de bainha com localização intracraniana em cães foram raramente relatados (Pumarola et al., 1996; Ottinger et al., 2009). A distinção entre tumores benignos ou malignos intracranianos não é tão relevante clinicamente, pois tumores com características citológicas benignas, que não possuem capacidade de metástase, podem ser uma ameaça à vida devido à infiltração no sistema nervoso. Os sinais neurológicos dependem primariamente da localização do tumor no sistema nervoso, do seu tamanho e da sua taxa de crescimento (Morris e Dobson, 2001; LeCouter e Withrow; 2007).

As neoplasias orbitárias são incomuns e usualmente provocam exoftalmia e diminuição ou ausência da retropulsão do bulbo. Dependendo da localização e extensão, pode ocorrer deslocamento do globo. A protusão da terceira pálpebra é geralmente evidente e, na maioria dos casos, a visão está mantida (Peiffer e Simons, 2002; Gelatt, 2003). Em um estudo que descreveu 25 casos de animais com tumor retrobulbar, a exoftalmia unilateral foi o sinal clínico mais frequente (84\%), seguida da hiperemia conjuntival $(40 \%)$ e da protusão da terceira pálpebra (28\%) (Attali-Soussay et al., 2001). Nos casos em que há infecção secundária da órbita, pode-se observar uveíte (Peiffer e Simons, 2002; Gelatt, 2003). A compressão retrobulbar por neoplasia, abscesso ou trauma pode lesionar a inervação simpática, gerando a síndrome de Horner pós-ganglionar (Broek, 1987). Esses sinais clínicos foram observados no paciente do presente relato. Outros sinais que podem ser encontrados são quemose, eritema conjuntival, edema periorbital, dor ao abrir a boca, cegueira central e acúmulo de fluido subretinal (Boston, 2010).
O tumor infiltrativo na região selar do crânio possivelmente ocasionou o aumento da pressão intracraniana que, consequentemente, levou ao reflexo de Cushing, resultando em bradicardia, com o objetivo de manter o fluxo sanguíneo cerebral constante (Bonafine, 2003). Adicionalmente, a compressão do globo ocular e, consequentemente, a do nervo vago induziram o reflexo oculocardíaco e contribuíram para a diminuição na frequência cardíaca.

A realização de radiografias simples do crânio auxilia pouco na detecção de neoplasias intracranianas, mas pode ser útil no diagnóstico de tumores que envolvem a cavidade nasal e os seios paranasais, pois pode haver lise óssea da órbita ou da cavidade nasal (Morris e Dobson, 2001; Boston, 2010). Exames de imagem podem ser utilizados para avaliação oftálmica e a escolha do método empregado depende da disponibilidade, do custo e da área anatômica de interesse. Em geral, a radiografia e a tomografia computadorizada são preferidas para avaliação da órbita óssea, enquanto a ressonância magnética e a ultrassonografia são mais indicadas para avaliação de tecidos moles, e a ressonância magnética é o exame de escolha para avaliação do SNC (Wilkie e Willis, 2002). A ultrassonografia é um exame complementar facilmente disponível e útil para a localização da massa retrobulbar (Gelatt, 2003; Boston, 2010). No paciente do presente relato, optou-se por realizar radiografia e ultrassonografia da região retrobulbar devido à disponibilidade dos exames e eles permitiram descartar destruição óssea da região, porém não foi possível delimitar o tamanho e a extensão da massa. Radiografias torácicas e ultrassonografia abdominal são indicadas para descartar possíveis metástases (Morris e Dobson, 2001; Gelatt, 2003).

Em seres humanos, os tumores de bainha orbitários são raros e, quando ocorrem, são geralmente de crescimento lento, não invasivos, não encapsulados. A morfologia varia de circular a oval, apresentando efeito mínimo nas estruturas orbitais adjacentes. Devido a essas características, a cirurgia é indicada nos seres humanos e possui bons resultados (Rootman et al., 1982; Huang et al., 2003; Rawlings, 2007).

Ao exame histológico, a descrição clássica dos tumores de bainha perineural é referida como padrão tipo Antoni A, que é caracterizado pela 
presença de células fusiformes altamente arranjadas em paliçadas, e tipo Antoni B, que possui menor celularidade e organização (Kuamura et al., 1998; Stoica et al., 2001; Diaz et al., 2007; Viott et al., 2007), embora em cães esses padrões não sejam predominantes como em seres humanos (Silva et al., 2012).

Tumores malignos da bainha perineural geralmente não apresentam cápsula e têm aspecto infiltrativo. Microscopicamente, são menos diferenciados, pleomórficos, constituídos de células anaplásicas que infiltram os tecidos adjacentes ou fazem, mais raramente, metástase para linfonodos ou pulmão (Stoica et al., 2001; LeCouter e Withrow, 2007; Tammenhain et al., 2008).

Os tumores de nervos periféricos são geralmente positivos para vimentina e S100. A imunoexpressão inconsistente para S100 indica caráter de malignidade (Pumarola et al., 1996; Viott et al., 2007; Silva et al., 2012). Esses neoplasmas podem ter aspectos morfológicos variados e, dessa forma, para a conclusão diagnóstica, foi necessário o uso concomitante da marcação de GFAP. Assim, neste caso, os achados macro e microscópicos de invasão dos tecidos adjacentes, áreas de necrose e baixa imunoexpressão para S100 e GFAP foram necessários para caracterizar a malignidade da neoplasia. Devido à infiltração da região, a possível origem da neoplasia é o III par de nervos cranianos e não o nervo óptico, já que do quiasma ao globo não havia alterações.

A exploração cirúrgica para realização de biópsia é essencial para o diagnóstico e, em alguns cães, é o único método que pode confirmar a presença desta neoplasia (LeCouter e Withrow, 2007; Tammenhain et al., 2008). Como no presente caso não foi possível avaliar a extensão da massa pelos exames de imagem realizados, foi indicada a exploração cirúrgica e, caso não fosse possível retirar toda a massa sem a realização de craniotomia, esta seria removida parcialmente para aliviar alguns sinais clínicos e para fornecer material para o diagnóstico histológico. Contudo, dependendo da infiltração dos tecidos adjacentes, seria indicada a eutanásia.

O tratamento de eleição é a excisão cirúrgica, no entanto a cirurgia da base do crânio é pouco comum e a completa excisão da massa é impossível na maioria dos pacientes, devido à característica infiltrativa da neoplasia (Diaz et al., 2000; Voll et al., 2006; LeCouter e Withrow, 2007; Araújo et al., 2008; Tammenhain et al., 2008; Fernandez e Bernardini, 2010; Silva et al., 2012). A quimioterapia com ciclofosfamida, vincristina e doxorrubicina é recomendada, considerando que os efeitos são apenas paliativos (Diaz et al., 2000; LeCouter e Withrow, 2007). A radioterapia e a quimioterapia apresentam benefício mínimo (Diaz et al., 2000; LeCouter e Withrow, 2007; Araújo et al., 2008; Silva et al., 2012).

O prognóstico dos tumores de nervos periféricos é pobre e a recorrência pós-cirúrgica é frequente (Voll et al., 2006; LeCouter e Withrow, 2007; Tammenhain et al., 2008; Fernandez e Bernardini, 2010; Silva et al., 2012). No paciente relatado, o prognóstico foi ruim, tanto pela localização como pelas sequelas devido ao provável aumento da pressão intracraniana. Estima-se que até $72 \%$ dos casos apresentam recidiva após a ressecção da neoplasia (Voll et al., 2006). Têm sido descritas metástases no pulmão após o procedimento cirúrgico (LeCouter e Withrow, 2007).

\section{CONCLUSÃO}

Em cães com exoftalmia e síndrome de Horner, a neoplasia retrobulbar é um diagnóstico diferencial importante, sendo o tumor da bainha perineural uma das etiologias. Quando o paciente apresenta bradicardia, além do reflexo oculocardíaco, deve-se pensar também em extensão da massa para o interior do crânio causando o aumento da PIC. No presente relato, o paciente apresentava neoplasia retrobulbar $\mathrm{e}$ intracraniana com origem em nervos cranianos, sem acometimento do nervo óptico e com possível origem no III par de nervos cranianos.

\section{REFERÊNCIAS}

ARAUJO, B.M.; KEMPER, B.; FIGUEIREDO, M.L et al. Schwannoma do plexo braquial em cão. Med. Vet., v.2, p.45-49, 2008.

ATTALI-SOUSSAY, K.; JEGOU, J.P.; CLERC, B. Retrobulbar tumors in dogs and cats: 25 cases. Vet. Ophtalmol., v.4, p.19-27, 2001. 
BONAFINE, R. Emergencias Neurologicas. In: F. PELLEGRINO; A. SURANITI; L. GARIBALDI. $E l$ Libro de Neurologia para la Practica Clinica. Belo Horizonte: Inter-Médica, 2003. p.407-416.

BOSTON, S.E. Craniectomy and orbitectimy in dogs and cats. Can. Vet. J. v.51, p.537-540, 2010.

BROEK, A.H.M. Horner's syndrome in cats and dogs: a review. J. Small Anim. Pract., 28, 929-940, 1987.

CHIJIWA, K.; UCHIDA, K.; TATEYAMA, S. Immunohistichemical evaluation of canine peripheral nerve sheath tumors and other soft tissue sarcomas. Vet. Pathol,. v.41, p.307-318, 2004.

DIAZ, M.G.R.; NOVA, L.A.C.; FLORES, R.C. Schwannoma en la region pelvic de un perro de raza Doberman. Vet. Mex., v.31, p.169-172, 2000.

FERNANDEZ, V.L.; BERNARDINI, M. Neoplasias e síndromes paraneoplásicas. In: Neurologia em cães e gatos. Belo Horizonte: Medvet, 2010. p.269298.

GELATT, K.N. Doenças e cirurgia da órbita do cão. In: K.N. GELATT, K.N. Manual de Oftalmologia Veterinária. São Paulo: Manole, 2003. p.27-46.

GOLDSCHMIDT, M.H.; HENDRICK, M.J. Tumors of the skin and soft tissues. In: MEUTEN, J. (Ed.). Tumors in domestic animals. 4.ed. New Jersey Wiley Blackwell 2002p. 95-96.

HUANG, W.T.; CHEN, W.; HSU, H.C. et al. Retrobulbar cellular schwannoma. Two cases report and review of literature. Pathol. Res. Pract., v.199, p.171-174, 2003.

JONES, B.R.; ALLEY, M.R.; JOHNSTONE, A.C.; JONES, J.M.; CAHILL, J.I. et al. Nerve sheath tumours in the dog and cat. N. Z. Vet. J., v.43, p.190196, 1995.

KUAMURA, M.; YAMATE, J.; KOTANI, T. et al. Canine peripheral nerve sheath tumor with eosinophilic cytoplasmic globules. Vet. Pathol., v.35, p.223-226, 1998.

LeCOUTER, R.A.; WITHROW, S.J. Tumors of the nervous system. In: WITHROW, S.J.; VAIL, D.M. Small animal oncology. 4.ed. [London]: Blackwell, 2007, p.659-680.

MORRIS, J.; DOBSON, J. Nervous System. In: Small Animal Oncology. [London]: Blackwell, 2001. p.192-203.

OTTINGER, T.; LINDBERG, R.; EKMAN, S. Malignant acoustic schwannoma in a dog. J. Vet. Diag. Inest., v.21, p.129-132, 2009.
PEIFFER, R.L.; SIMONS, K.B. Orbital tumors. In: Ocular tumors in animals and humans. Iowa: State Press, 2002. p.3-23.

PUMAROLA, M.; ANOR, S.; BORRAS, D.; FERRER, I. Malignant epithelioid Schwannoma affecting the trigeminal nerve of a dog. Vet. Pathol., v.33, p.434-436, 1996.

RAWLINGS, N.G.; BROWNSTEIN, S.; ROBINSON, J.W.; JORDAN, D.R. Orbital schwannoma: histopathologic correlation with magnetic resonance imaging. Can. J. Ophthal., v.42, p.326-328, 2007.

RODRIGUEZ, F.J.; FOLPE, A. L.; GIANNINI, C.; PERRY, A. Pathology of peripheral nerve sheath tumors: diagnostic overview and update on selected diagnostic problems. Acta Neuropathol., v.123, p.295319,2013

ROOTMAN, J.; GOLDBERG, C.; ROBERTSON, W. Primary orbital schwannomas. Br. J. Ophthalmol., v.66, p.194-204, 1982.

SILVA, E.O.; ZANONI, F.P.; FERIOLI, R.B. et al. Tumor maligno da bainha do nervo periférico envolvendo raízes nervosas do terceiro segmento medular lombar em um cão. Semin. Ciênc. Agrár., v.33, p.2397-2402, 2012.

STOICA, G.; TASCA, S.I.; KIM, H.T. Point mutation of neu oncogene in animal peripheral nerve sheath tumors. Vet. Pathol., v.38, p.679-688, 2001.

TAMMENHAIN, B.; OBLADEN, A.; WERNER, J.; FARIAS, M. Schwannoma maligno em cão - relato de caso. Medvep Rev. Cient. Med. Vet. Pequenos Anim. Anim. Estimação, v.6, p.75-79, 2008.

VIOTT, A.M.; RAMOS, M.A.; INKELMANN, M.A. et al. Aspectos histoquímicos e imunohistoquímicos nos neoplasmas do sistema nervoso periférico. Arq. Bras. Med. Vet. Zootec., v.59, p.1145-1153, 2007.

VOLL, J.; VOLL, R.; GAIGA, L. et al. Exerese de schwannoma por meio da técnica de hemilaminectomia dorsolateral cervical cranial. Acta Scient. Vet., v.34, p.183-186, 2006.

WILKIE, D.A.; WILLIS, M. Ophthalmic imaging. In: RISS, R.C. Small animal ophthalmology secrets. Rio de Janeiro: Elsevier, 2002. p.13-17.

ZACHARY, J.F. Sistema nervoso. In. M.D. McGAVIN, M.D.; ZACHARYF, J.F. Bases da patologia em veterinária. 4.ed. Philadelphia: Mosby Elsevier, 2009. p.833-972. 